\title{
Neoadjuvant chemoradiotherapy for patients with unresectable radically locally advanced colon cancer: a potential improvement to overall survival and decrease to multivisceral resection
}

Yan Yuan ${ }^{1,2+}$, Wei-Wei Xiao ${ }^{1,2+}$, Wei-Hao Xie ${ }^{1,2+}$, Pei-Qiang Cai ${ }^{1,3}$, Qiao-Xuan Wang ${ }^{1,2}$, Hui Chang ${ }^{1,2}$,

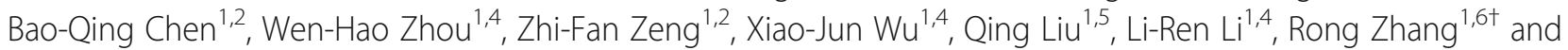
Yuan-Hong Gao ${ }^{1,2^{*}+}$

\begin{abstract}
Background: The management of unresectable locally advanced colon cancer (LACC) remains controversial, as resection is not feasible. The goal of this study was to evaluate the treatment outcomes and toxicity of neoadjuvant chemoradiotherapy (NACRT) followed with surgery and adjuvant chemotherapy in patients with unresectable radically LACC.

Methods: We included patients who were diagnosed at our institution, 2010-2018. The neoadjuvant regimen consisted of radiotherapy and capecitabine/ 5-fluorouracil-based chemotherapy.

Results: One hundred patients were identified. The median follow-up time was 32 months. The R0 resection rate, adjusted nonmultivisceral resection rate and bladder preservation rate were 83.0, 43.0 and 83.3\%, respectively. The pCR and clinical-downstaging rates were 18, and $81.0 \% \%$, respectively. The 3-year PFS and OS rates for all patients were 68.6 and $82.1 \%$, respectively. Seventeen patients developed grade 3-4 myelosuppression, which was the most common adverse event observed after NACRT. Tumor perforation occurred in 3 patients during NACRT. The incidence of grade 3-4 surgery-related complications was 7.0\%. Postoperative anastomotic leakage was observed in 3 patients.

(Continued on next page)
\end{abstract}

\footnotetext{
* Correspondence: gaoyh@sysucc.org.cn

†Yan Yuan, Wei-Wei Xiao, Wei-Hao Xie, Rong Zhang and Yuan-Hong Gao contributed equally to this work.

${ }^{1}$ State Key Laboratory of Oncology in South China, Collaborative Innovation Center for Cancer Medicine, Guangzhou, China

2Department of Radiation Oncology, Sun Yat-sen University Cancer Center, Guangzhou, China

Full list of author information is available at the end of the article
}

(c) The Author(s). 2021 Open Access This article is licensed under a Creative Commons Attribution 4.0 International License, which permits use, sharing, adaptation, distribution and reproduction in any medium or format, as long as you give appropriate credit to the original author(s) and the source, provide a link to the Creative Commons licence, and indicate if changes were made. The images or other third party material in this article are included in the article's Creative Commons licence, unless indicated otherwise in a credit line to the material. If material is not included in the article's Creative Commons licence and your intended use is not permitted by statutory regulation or exceeds the permitted use, you will need to obtain permission directly from the copyright holder. To view a copy of this licence, visit http://creativecommons.org/licenses/by/4.0/ The Creative Commons Public Domain Dedication waiver (http://creativecommons.org/publicdomain/zero/1.0/) applies to the data made available in this article, unless otherwise stated in a credit line to the data. 
(Continued from previous page)

Conclusions: NACRT followed by surgery was feasible and safe for selected patients with LACC, and can be used as a conversion treatment to achieve satisfactory downstaging, long-term survival and quality of life, with acceptable toxicities.

Keywords: Locally advanced colon cancer, Neoadjuvant chemoradiotherapy, Organ preservation, Pathological complete response

\section{Introduction}

Colon cancer is one of the most common cancers worldwide and accounted for approximately $6.1 \%$ of newly diagnosed cancers and $5.8 \%$ cancer-related deaths in 2018 [1]. Approximately 26\% of patients with colon cancer present with a locally advanced disease [2]. In patients with locally advanced colon cancer, including patients with high-risk stage II or stage III disease, surgery and adjuvant chemotherapy are the standard treatments [3]. However, R0 resection is unable to be achieved in some patients with T4b, M0 or N2, M0 disease, even after multivisceral resection (MVR) [4]. Incomplete resection has not been shown to be beneficial [5]. Therefore, converting unresectable LACC to achieve radical resection may be essential to improve the quality of life and prolong the survival time of patients.

Neoadjuvant chemoradiotherapy (NACRT) has been well established as the standard therapy for locally advanced rectal cancer (LARC), and is related to a survival benefit and organ preservation [6]. The pathogenesis of colon and rectal cancer is similar. Thus, NACRT is worthy of investigation in patients with unresectable LACC. Actually, several reports with a small sample size have evaluated NACRT followed by radical surgery for LACC [7]. We also previously published several studies with small sample sizes $[8,9]$. Results suggest that patients with LACC may benefit from NACRT. In the present study, we described the results of the administration of NACRT to patients with LACC over the last decade at a comprehensive cancer center.

\section{Methods}

\section{Patient population and staging system}

The study was designed to evaluate the value of the NACRT for patients with unresectable LACC. This observational study was approved by our institutional medical ethics committee (B2020-063-01). One hundred eligible patients were identified who were diagnosed at our hospital from November 1, 2010 to June 31, 2018. Patients were selected to undergo NACRT on a case-by-case basis through a consultation with a multidisciplinary team (MDT). The pretreatment evaluation, the definition of unresectability and key exclusion criteria were described in our previous reports $[8,9]$. Patients with LACC (defined as the primary tumor having an inferior margin $\geq 15$ $\mathrm{cm}$ from the anal verge, as determined by colonoscopy) were candidates for NACRT if they met the criteria listed in the Fig. 1a. Patients' medical records were reviewed and demographic, oncological, and pathological information was collected. A Charlson comorbidity index score was generated for each patient after a review of the medical history [10]. All patients have signed the informed consent form before treatment.

The pretreatment clinical stage of all patients treated before 2017 were re-evaluated according to the 8th edition of the Union for American Joint Cancer Committee (AJCC) TNM staging system. Then the 8th edition of TNM staging was used for clinical staging in patients who were diagnosed after 2017. Patients with peritoneal carcinomatosis classified as M1c were excluded from this study. Chest/abdomen/ pelvic computed tomography (CT), pelvic magnetic resonance image (MRI), serum chemical profile including carcinoembryonic antigen (CEA), and considering colonoscopy were performed after NACRT to assess the response of tumors.

\section{Treatments and follow-up}

Chemotherapy and radiotherapy, and surgery were described in detail in our previous reports [8]. Standard complete mesocolic excision (CME) would be performed after NACRT. When tumor infiltration or adhesion to the adjacent organs was detected intraoperatively, MVR was required. Radial margins were evaluated based on the pathological review principles of the NCCN guidelines. The formalin-fixed paraffin-embedded blocks of surgical samples from a portion of patients were tested for the MMR status using immunohistochemical staining for MLH1, MSH2, MSH6, and PMS2. Acute adverse events that occurred during or 30 days after NACRT were graded according to the Common Terminology Criteria for Adverse Events (CTCAE) (version 4.03). Postoperative complications were assessed according to the Clavien-Dindo classification [11]. 
$\mathbf{A}$

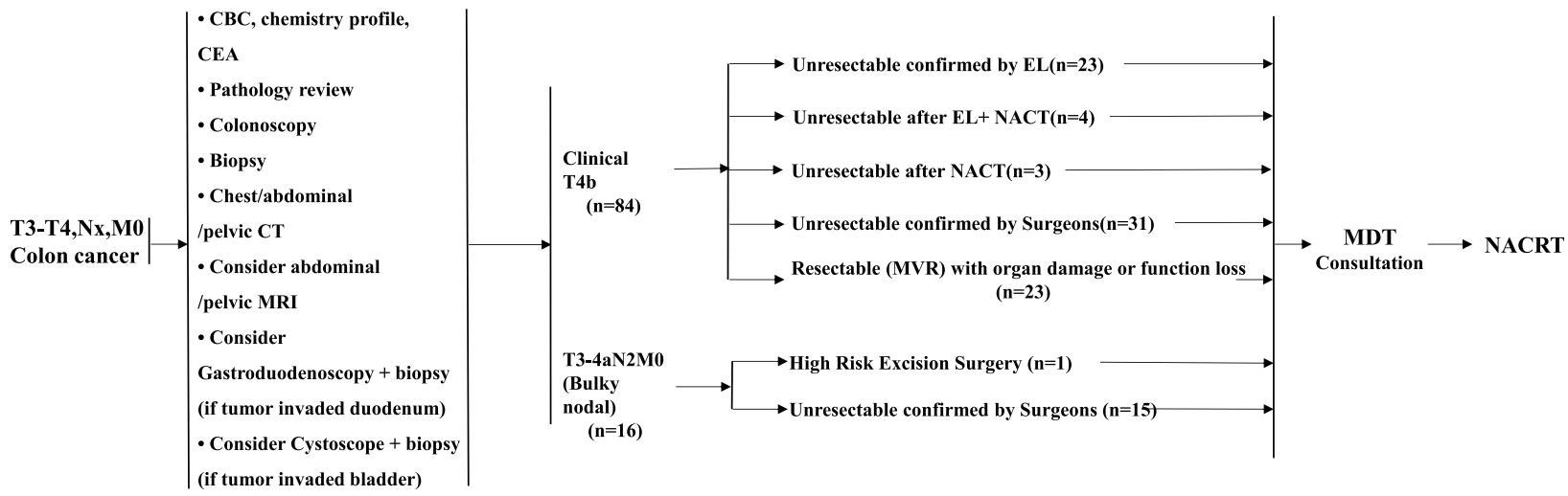

B

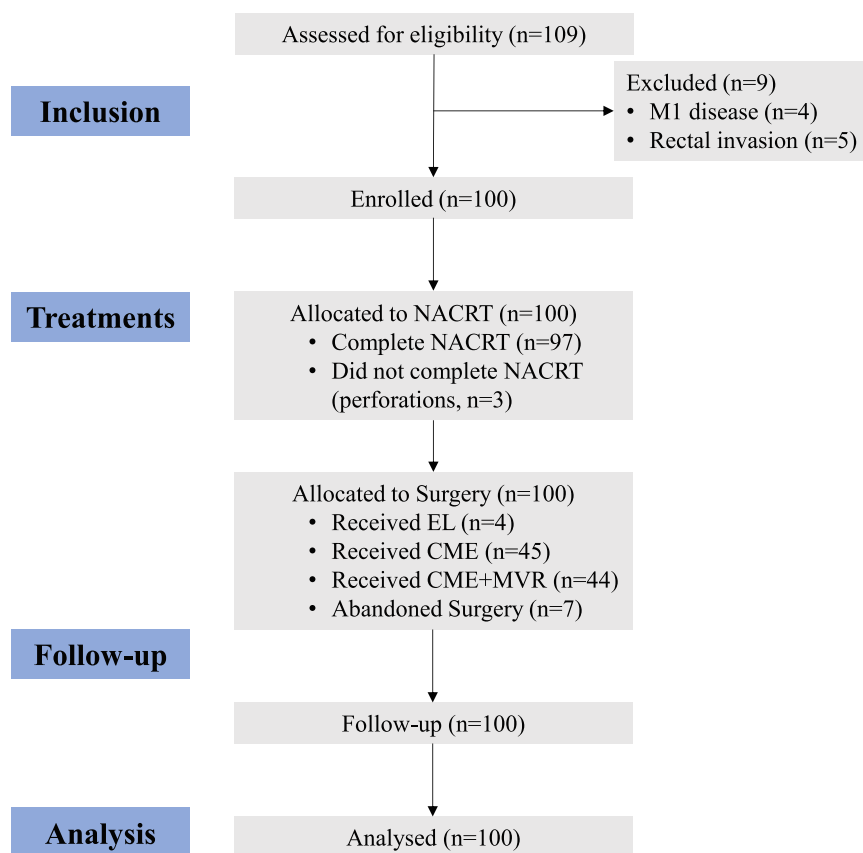

Fig. 1 Flowchart of diagnosis of unresectable LACC (a) and Study profile (b). NACRT, Neoadjuvant chemoradiotherapy; EL, exploratory laparotomy; CME, complete mesocolic excision; MVR, multivisceral resection

Follow-up visits were performed by a multidisciplinary team every 3 months in the first 2 years after treatment, every 6 months in the following 3 years, and then yearly thereafter. Afterwards the patients were followed by an outpatient interview or household registration system. The last follow-up time point was December 31, 2019.

\section{Statistical analysis}

Descriptive statistics were used to report patient, tumor, and treatment characteristics. Continuous data are presented as medians with interquartile ranges (IQRs).
Categorical data are presented as numbers with percentages (\%). Progression-free survival (PFS) was calculated from the initial diagnosis to the first disease progression or death. Overall survival (OS) was calculated from the diagnosis to the date of death. Disease-free survival (DFS) was calculated from the initial diagnosis to the first disease progression or death in the R0 group. The local recurrence (LR) rate was calculated in the R0 group. The distant metastasis (DM) rate was evaluated in all patients. The survival analysis was performed using $\mathrm{R}$ version 3.6.0. The packages 'survival' and 'survminer' were used for the survival analysis. Survival curves were 
also constructed by using $\mathrm{R}$ version 3.6.0. The multicollinearity regressions, the correlation matrix analysis, the Cox regression analysis, the Cox proportional hazard regression model were performed using STATA software (version 15). The multicollinearity regressions and a correlation matrix analysis were used to calculate the correlations between variables. Univariate and multivariate analyses were conducted to identify prognostic factors. A Cox regression analysis was used for univariate and multivariate analyses. The Cox proportional hazard regression model was used to estimate the hazard ratio (and corresponding 95\% confidence interval $[\mathrm{CI}]$ ) for each of the potential risk factors. A two-sided $P$-value $<0.05$ was considered statistically significant.

\section{Results}

\section{Characteristics and compliance}

The study profile is shown in Fig. 1b. Patient characteristics, tumor staging and treatment details of the 100 patients with unresectable LACC are listed in Table 1. The pathological type of all patients was adenocarcinoma. All patients underwent colonoscopy and pathological biopsy at diagnosis. Eighty-four patients $(84.0 \%)$ were diagnosed with stage cT4b tumors. The most common tumor location was the sigmoid colon $(60 / 100,60.0 \%)$, and bladder was the most commonly invaded organ $(42 / 100,42.0 \%)$. Fourteen $(14 / 100,14.0 \%)$ of these patients had a bladder fistula caused by tumor infiltration prior to the treatments. Meanwhile, 19 (19/100, 19.0\%) patients had an intestinal obstruction at diagnosis. All patients received NACRT after the remission of the obstruction. Prophylactic enterostomy was performed in 27 (27/ 100, 27.0\%) patients.

Neoadjuvant chemotherapy was a capecitabine/ 5FUbased regimen with a median cycles number of 4 (IQR 3-4). The sketching methods of GTV and CTV are described in our previous two articles $[8,9]$. The dose of GTV is 46-54Gy/23-27 fractions. The dose of CTV is 41.4-46 Gy/23-27 fractions. Three patients developed colon cancer perforation during NACRT. Ninety-seven patients successfully received the allocated NACRT. 93 (93/100, 93.0\%) patients underwent surgical treatment, and the other $7(7 / 100,7.0 \%)$ patients abandoned further surgical treatment after NACRT with treatment details shown in Supplementary Table 1.

\section{Short-term efficacy}

Among all patients receiving NACRT, 93 (93/100, 93.0\%) patients underwent surgery with the goal of a radical operation (Supplementary Figure 1). The details of operations and pathological findings from the 93 patients are presented in the Table 2 . In this cohort study,
$89.25 \%(83 / 93)$ of the 93 patients who underwent surgery reached $\mathrm{R} 0$, and $81.0 \%(81 / 100)$ patients achieved downstaging after NACRT. The pCR rate was $18.0 \%$ $(18 / 100)$.

Seventy $(83.3 \%, 70 / 84)$ patients with cT4b diseases achieved R0 resection. 34.5\% (29/84) patients underwent CME without MVR and 48.8\% (41/84) patients required $C M E$ with $M V R$. The changes in the imaging features of cT4b patients after NACRT are shown in Fig. 2.

Among the 42 patients with pretreatment bladder invasion (Supplementary Figure 2A), 2 (2/42, 4.8\%) and 22 $(22 / 42,52.4 \%)$ patients underwent total cystectomy and partial cystectomy respectively. $13(13 / 42,31.0 \%)$ patients did not have bladder resection. Five (5/42, 11.9\%) patients did not undergo cystectomy due to unresectability or personal reasons. Therefore, totally $83.3 \%$ (35/ 42) patients retained bladder function.

In addition, the small intestine is the second most common site of adjacent organ invasion in patients with LACC (Supplementary Figure 2B). Nine (9/26, 34.6\%) patients with $\mathrm{R} 0$ resection avoided small intestine resection. 7 (7/26, 26.9\%) patients underwent small bowel resection and $4(4 / 26,15.4 \%)$ patients had a Whipple's procedure. Six $(6 / 26,23.1 \%)$ patients did not received radical surgery because the conversion therapy failed or for personal reasons.

\section{Long-term survival}

The median follow-up period of surviving patients was 32 (IQR 24-55) months in the entire group. Using the public security household registration system, we inquired about the survival outcomes of 6 patients who were lost medical follow-up. None of the patients were lost to follow-up.

The estimated PFS rate at 3 years was $68.6 \%$ for the whole group (Fig. 3a). The estimated OS rate at 3 years was $82.1 \%$ (Fig. 3a). In this study, local control failed in $16(16 / 100,16.0 \%)$ patient, and DM occurred in 28 (28/ $100,28.0 \%)$ patients. Among the patients who underwent R0 surgery, the 3 -year PFS, OS, LR and DM rates were $74.0 \%$ (Fig. 3b), 89.6\% (Fig. 3c), 13.4 and $20.8 \%$, respectively. As expect, the LR rate and DM rate of 17 patients with non-R0 resection were disappointing. The cumulative 3 year PFS, OS, local progression, DM rates for this cohort were $38.1,45.8,71.9$, and $41.2 \%$, respectively.

In the univariate analysis (Supplementary Table 3), low differentiation, non-R0 resection, ypT stage (ypT4aT4b) and advanced ypTNM stage (ypIIb-IIIc) were significantly associated with poor OS and PFS in the whole group (Supplementary Figure 3A-H), and a low KPS, low differentiation, and VLPNI (vascular or lymphatic or perineural invasion) positively were associated with a 
Table 1 Baseline pathological and clinical characteristics of patients

\begin{tabular}{|c|c|}
\hline & No. (\%) \\
\hline Age, median, years & $54(43-63)$ \\
\hline$\leq 65$ & $84(84.0 \%)$ \\
\hline$>65$ & $16(16.0 \%)$ \\
\hline \multicolumn{2}{|l|}{ Sex } \\
\hline Male & 73 (73.0\%) \\
\hline Female & $27(27.0 \%)$ \\
\hline \multicolumn{2}{|l|}{ KPS } \\
\hline 90 & 78 (78.0\%) \\
\hline 80 & $22(22.0 \%)$ \\
\hline BMI, median, & $21.4(19.5-23.6)$ \\
\hline Primary tumor length, median, $\mathrm{cm}$ & $7.4(1.7-8.9)$ \\
\hline \multicolumn{2}{|l|}{ Primary tumor location } \\
\hline Sigmoid colon & $60(60.0 \%)$ \\
\hline Descending colon & $3(3.0 \%)$ \\
\hline Transverse colon & $10(10.0 \%)$ \\
\hline Ascending colon & $25(25.0 \%)$ \\
\hline Ileocecus & $2(2.0 \%)$ \\
\hline \multicolumn{2}{|l|}{ Tumor differentiation } \\
\hline High & $20(20.0 \%)$ \\
\hline Moderate & $65(65.0 \%)$ \\
\hline Low & $15(15.0 \%)$ \\
\hline CEA pre-CRT, median, ng/ml & $6.1(3.0-21.3)$ \\
\hline \multicolumn{2}{|l|}{ Complication } \\
\hline No & $56(56.0 \%)$ \\
\hline Yes & $44(44.0 \%)$ \\
\hline \multicolumn{2}{|l|}{ cT stage } \\
\hline T3 & $4(4.0 \%)$ \\
\hline T4a & $12(12.0 \%)$ \\
\hline T4b & $84(84.0 \%)$ \\
\hline \multicolumn{2}{|l|}{ cN stage } \\
\hline No & $1(1.0 \%)$ \\
\hline N1 & $36(36.0 \%)$ \\
\hline N2 & $63(63.0 \%)$ \\
\hline \multicolumn{2}{|l|}{ Clinical stage } \\
\hline IIC & $1(1.0 \%)$ \\
\hline IIIB & $16(16.0 \%)$ \\
\hline IIIC & $83(83.0 \%)$ \\
\hline \multicolumn{2}{|l|}{ Involved organ } \\
\hline Bladder & $42(42.0 \%)$ \\
\hline Ureter & $12(12.0 \%)$ \\
\hline Renal and perirenal fat, prerenal space & $6(6.0 \%)$ \\
\hline Pelvic wall & $10(10.0 \%)$ \\
\hline Presacral space & $2(2.0 \%)$ \\
\hline Abdominal wall & $11(11.0 \%)$ \\
\hline
\end{tabular}


Table 1 Baseline pathological and clinical characteristics of patients (Continued)

\begin{tabular}{|c|c|}
\hline & No. (\%) \\
\hline Mesentery & $2(2.0 \%)$ \\
\hline Great vessel & $8(8.0 \%)$ \\
\hline Small intestine & $26(26.0 \%)$ \\
\hline Greater omentum & $5(5.0 \%)$ \\
\hline Gallbladder & $6(6.0 \%)$ \\
\hline Liver & $9(9.0 \%)$ \\
\hline Appendix & $1(1.0 \%)$ \\
\hline Pancreas & $2(2.0 \%)$ \\
\hline Uterus & $6(6.0 \%)$ \\
\hline Vagina & $1(1.0 \%)$ \\
\hline Seminal vesicle gland & $3(3.0 \%)$ \\
\hline Vas deferens & $2(2.0 \%)$ \\
\hline Iliopsoas muscle & $1(1.0 \%)$ \\
\hline \multicolumn{2}{|l|}{ Bladder fistula/perforation } \\
\hline Yes & $14(14.0 \%)$ \\
\hline No & $86(86.0 \%)$ \\
\hline \multicolumn{2}{|l|}{ Intestinal obstruction } \\
\hline Yes & 19 (19.0\%) \\
\hline No & $81(81.0 \%)$ \\
\hline \multicolumn{2}{|l|}{ Family history } \\
\hline Yes & $20(20.0 \%)$ \\
\hline No & $80(80.0 \%)$ \\
\hline \multicolumn{2}{|c|}{ Charlson Comorbidity Score } \\
\hline 0 & $82(82.0 \%)$ \\
\hline 1 & $15(15.0 \%)$ \\
\hline 2 & $2(2.0 \%)$ \\
\hline 3 & $1(1.0 \%)$ \\
\hline \multicolumn{2}{|l|}{ MMR } \\
\hline dMMR & $14(22.6 \%)$ \\
\hline pMMR & $48(77.4 \%)$ \\
\hline Unknown & 38 \\
\hline
\end{tabular}

Abbreviations: KPS Karnofsky Performance Status, BMI Body Mass Index, CT stage Clinical T stage, cN stage Clinical N stage, MMR Mismatch repair phenotype

poor DFS in the R0 group (Supplementary Figure 3I-K). Both the results of multicollinearity regression (Supplementary Table 4.1) and the correlation matrix (Supplementary Table 4.2) identified strong correlations between two variables (ypT stage group and ypTNM stage group). Then we subjected the ypTNM stage group to the multivariate analysis. In the multivariate analysis, differentiation remained an independent prognostic factor for OS rates (Table 3). However, no difference in survival was observed between patients with different ypN stages, MMR status, genders, ages and nutritional statuses. A Low TRG score seems to be associated with the poor OS but failed to reach the significance
(Supplementary Figure 3M). We supposed that the number of each group was small to reach statistical difference.

The information about the treatment-related toxicities is shown in Table 4. Based on the CTCAE criteria ver. 4.03 , the most common grade 3 to 4 NACRT-related toxicities were myelosuppression, gastrointestinal (GI) toxicities and mucositis/dermatitis. The incidence rates were 17.0, 7.0, and 3.0\%, respectively. Eight patients developed an intestinal obstruction during NACRT. Seven patients developed diarrhea and abdominal pain in the process of radiotherapy. Only 3 patients did not complete the radiation course due to tumor rupture, of 
Table 2 Treatment outcomes of operations and pathological findings

\begin{tabular}{|c|c|}
\hline & No. (\%) \\
\hline CEA preoperative, median, $\mathrm{ng} / \mathrm{ml}$ & $2.4(1.5-4.1)$ \\
\hline \multicolumn{2}{|l|}{ Surgery situation } \\
\hline Ro & $83(89.2 \%)$ \\
\hline R2 & $6(6.5 \%)^{b}$ \\
\hline $\mathrm{EL}^{\mathrm{a}}$ & $4(4.3 \%)$ \\
\hline \multicolumn{2}{|l|}{ Downstage T } \\
\hline Yes & $65(69.9 \%)$ \\
\hline No & $28(30.1 \%)^{c}$ \\
\hline \multicolumn{2}{|l|}{ Downstage $\mathrm{N}$} \\
\hline Yes & $84(90.3 \%)$ \\
\hline No & $9(9.7 \%)^{c}$ \\
\hline \multicolumn{2}{|l|}{ Downstage } \\
\hline Yes & 81 (87.1\%) \\
\hline No & $12(12.9 \%)^{c}$ \\
\hline \multicolumn{2}{|l|}{ MVR } \\
\hline Yes & $44(47.3 \%)$ \\
\hline No & $49(52.7 \%)^{c}$ \\
\hline \multicolumn{2}{|l|}{ pCR } \\
\hline Yes & $18(19.4 \%)$ \\
\hline No & $75(80.6 \%)^{c}$ \\
\hline Resection Surgery-Radiotherapy interval Median, d (range) & $63.0(55-76.5)$ \\
\hline
\end{tabular}

which two patients underwent emergency surgery and one patient requested for supportive treatment instead of the operation as described before. No patients died during NACRT.

Among the 93 patients who underwent surgery, grade 3/4 Clavien-Dindo postsurgical complications were observed in 7 patients $(7 / 100,7.0 \%)$. In addition, 3 patients experienced anastomotic leakage after radical surgery, but completely recovered after the enterostomy or the repair of fistula.

\section{Discussion}

Worldwide, surgery is the cornerstone of curative treatment for the colorectal cancer. Radical resection is one of the most important predictors of LR and long-term survival in patients with stage III colon cancer [12]. Hence, a crucial question is whether patients with LACC are able to be converted from an unresectable status to resectable status with the goal of a cure. For locally unresectable radically or bulky nodal disease or clinical T4b colon cancer, neoadjuvant chemotherapy is recommended by NCCN guidelines [13]. In the present study, 7 patients with unresectable LACC underwent 3-5 courses of chemotherapy had stable diseases before NACRT and failed to convert to resection surgery. Therefore, more effective treatments are urgently needed.

Previously published studies have showed that NACR $\mathrm{T}$ is an effective choice for patient with unresectable LACC. The evidence of the effectiveness of NACRT for colon cancer is still accumulating. Between 2000 and 2010, several single or very small sample size case reports described the use of NACRT for colon cancer [14, 15]. Taylor et al. retrospectively analyzed 25 patients with LACC who were treated with en-bloc surgical resection with radiotherapy and chemotherapy. Those patients had a median survival of 38.2 months and a 5year survival rate of $49 \%$ [16]. Since 2010, a greater number of reports have investigated small sample sizes. The application of NACRT followed by MVR to 33 patients with primary locally advanced adherent colon cancer and 15 patients with locally recurrent adherent colon cancer patients both achieved high rates of R0 resection and excellent LR in studies published in 2012 and 2014, respectively, by Wong et al' team from Canada $[17,18]$ (Supplementary Table 2). In Taiwan, Chun- 
A
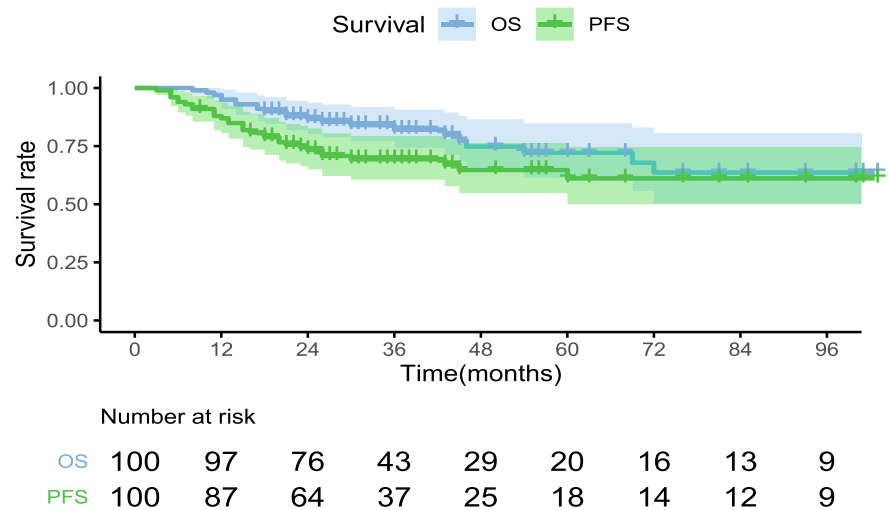

B

$\begin{array}{rlllllllll}\text { OS } & 100 & 97 & 76 & 43 & 29 & 20 & 16 & 13 & 9 \\ \text { PFS } & 100 & 87 & 64 & 37 & 25 & 18 & 14 & 12 & 9\end{array}$
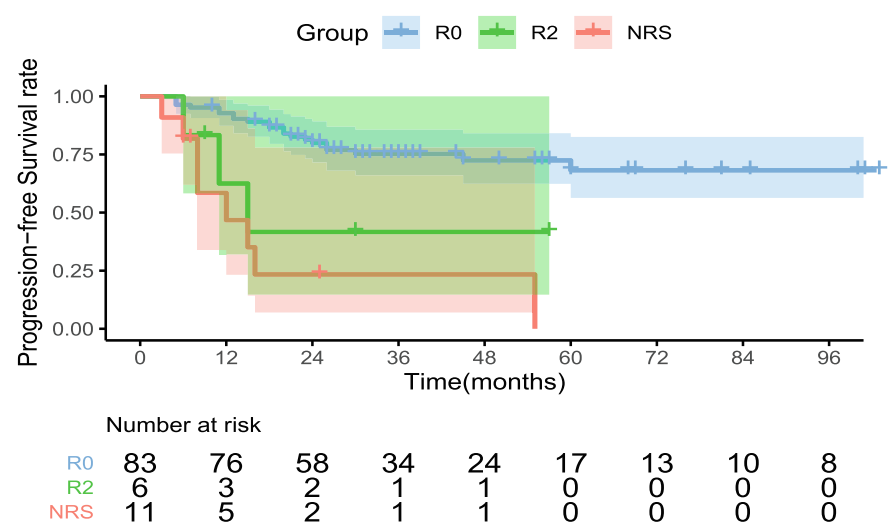

$\mathbf{C}$
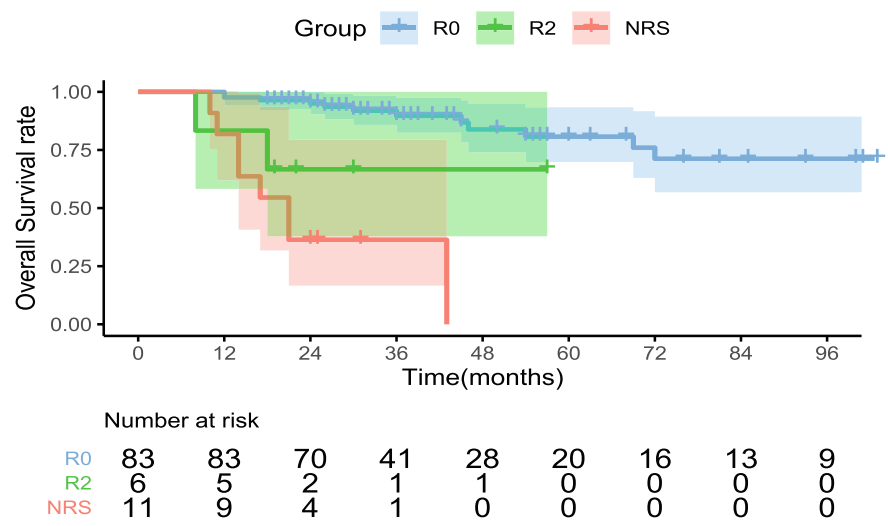

Fig. 2 Changes in the imaging features of patients with T4b tumors after neoadjuvant radiotherapy and chemotherapy. a Imaging figures presented ascending colon cancer with invasion of ileum (yellow arrow) and lymph node metastasis with invasion of parietal peritoneum (blue arrow). After NACRT, ascending colon cancer and lymph node metastasis were obviously smaller than before NACRT; b Imaging figures showed sigmoid colon cancer with invasion of bladder (brown arrow), small intestinal (yellow arrow) and peritoneum (blue arrow). Sigmoid colon cancer shrank significantly after NACRT

Ming Huang et al. delivered NACRT to 36 patients with potentially incomplete resection of LACC, as defined by the presence of a T3 tumor with extramural extension of $>5 \mathrm{~mm}$ or a $\mathrm{T} 4$ tumor diagnosed by imaging. Approximately $26.4 \%$ of patients achieved pCR and the 2-year estimated OS and DFS rates were 88.7 and $73.6 \%$, respectively [19].

Because the benefits of NACRT for colon cancer are not clear, the MDT of our cancer center began to explore the application of NACRT only in patients with 


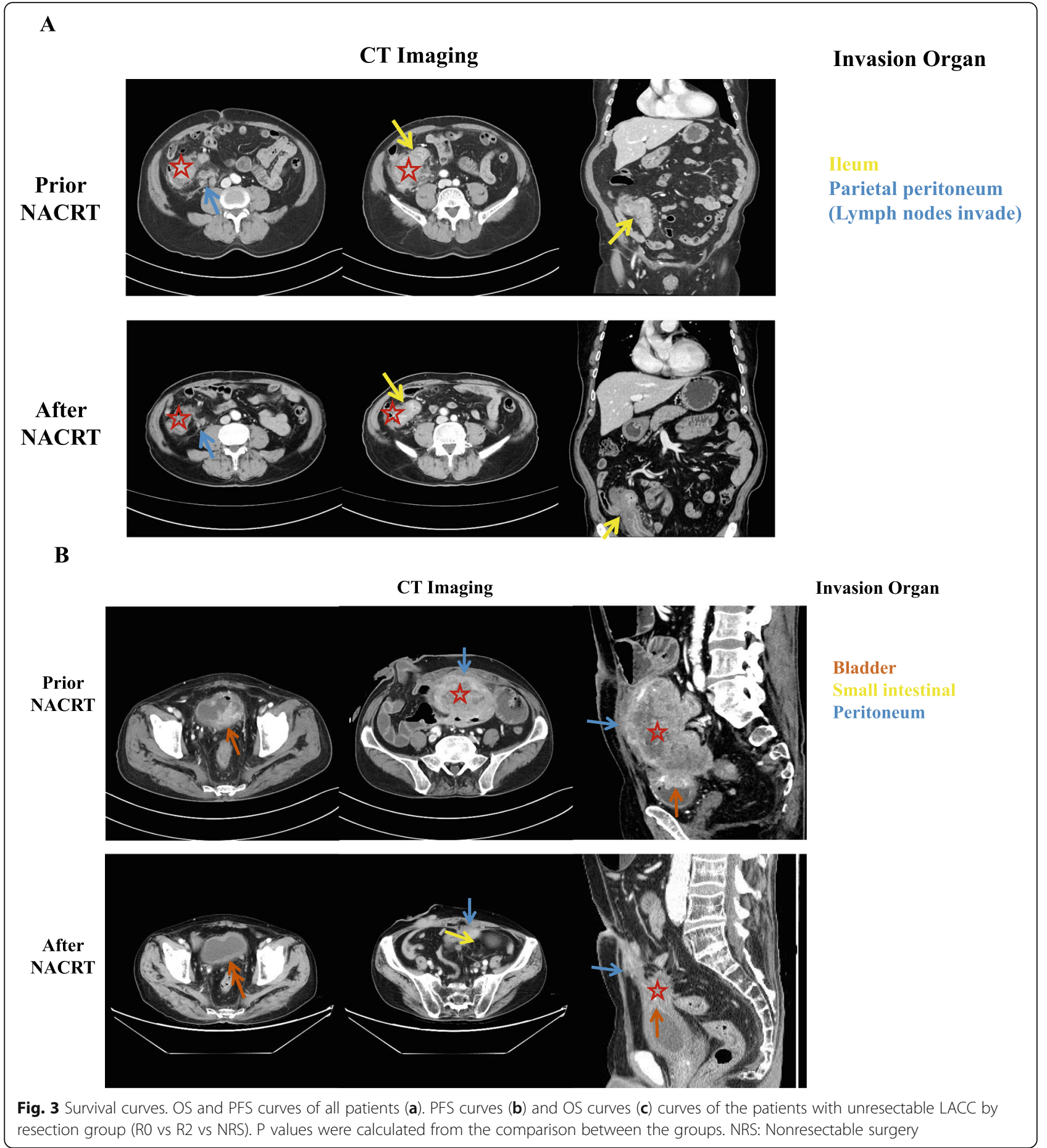

LACC. We have previously also reported the treatment outcomes of 21 and 60 patients with unresectable radically LACC in 2016 and 2018, respectively. The current report describes 100 patients enrolled from 2010 to 2018 and is the largest sample in which NACRT for LACC has been analyzed. Similar to previous studies, NACRT results in downstaging of tumor for patients with LACC, in which the pCR rate is $18 \%$. The low rate of $\mathrm{pCR}$ in the Canadian studies [17] may be due to the use of 5FU alone in NACRT, while we used double chemotherapy during NACRT, similar to the study by Huang CM et al. [19]. Doublet chemotherapy was well tolerated in the patients receiving NACRT for LACC and may contribute to better tumor regression, as observed in patients with 
Table 3 Multivariate Cox analysis of prognostic factors for OS, PFS, DFS. (OS: $p=0.0006, x 2=27 \cdot 27 ;$ PFS: $p=0.065, x 2=19 \cdot 35 ;$ DFS: $p=0.087, \times 2=9.62)$

\begin{tabular}{|c|c|c|c|c|c|c|}
\hline & \multicolumn{2}{|l|}{ OS } & \multicolumn{2}{|l|}{ PFS } & \multicolumn{2}{|l|}{ DFS } \\
\hline & HR $(95 \% \mathrm{Cl})$ & $p$ & HR $(95 \% \mathrm{Cl})$ & $p$ & HR $(95 \% \mathrm{Cl})$ & $p$ \\
\hline KPS $\left(80^{\mathrm{a}}\right.$ vs 90$)$ & .. &.$\cdot$ & $0.50(0.20-1.22)$ & 0.13 & $0.53(0.21-1.36)$ & 0.2 \\
\hline Complication (yes ${ }^{\mathrm{a}}$ vs no) & $1.70(0.54-5.41)$ & 0.37 & $1.56(0.67-3.64)$ & 0.3 & .. & .. \\
\hline Differentiation & .• & 0 & .. & 0.06 & $\cdot$. & 0.6 \\
\hline $\operatorname{High}^{\mathrm{a}}$ & 1 &.$\cdot$ & 1 &.. & 1 &.$\cdot$ \\
\hline Middle & $3.07(0.29-32.59)$ & 0.35 & $1.60(0.31-8.18)$ & 0.57 & $1.48(0.32-6.82)$ & 0.6 \\
\hline Low & $26.87(2.38-303.60)$ & 0.01 & $4.75(0.73-22.74)$ & 0.07 & $2.39(0.42-13.51)$ & 0.3 \\
\hline ypN stage ( $\mathrm{NO}^{\mathrm{a}}$ vs $\left.\mathrm{N} 1+\mathrm{N} 2\right)$ & $0.31(0.029-3.19)$ & 0.32 & $0.41(0.073-2.24)$ & 0.3 & .. & .. \\
\hline ypTNM stage (0-IIB ${ }^{a}$ vs IIC-IIIC) & $3.44(0.82-14.38)$ & 0.09 & $1.82(0.67-4.99)$ & 0.24 & $1.49(0.53-4.16)$ & 0.5 \\
\hline pCR (yes ${ }^{\mathrm{a}}$ vs non) & $0.40(0.053-2.99)$ & 0.37 & $1.18(0.29-4.84)$ & 0.81 & .. & $\cdot$. \\
\hline MVR (non ${ }^{a}$ vs yes) & $1.07(0.28-4.14)$ & 0.92 & $0.97(0.40-2.36)$ & 0.95 &.. &.. \\
\hline VLPNI (negative ${ }^{a}$ vs positive) & $3.61(0.84-15.54)$ & 0.09 & $2.16(0.77-6.02)$ & 0.14 & $2.01(0.64-6.37)$ & 0.2 \\
\hline $\mathrm{R} 0$ resection (non- $\mathrm{R}^{\mathrm{a}}$ vs $\mathrm{R} 0$ ) & $0.21(0.030-1.48)$ & 0.12 & $0.60(0.12-3.08)$ & 0.54 &.. &.$\cdot$ \\
\hline
\end{tabular}

Abbreviations: KPS Karnofsky Performance Status, cT stage Clinical T stage, MVR Multivisceral resection, $p C R$ Pathologic complete remission, VLPNI Vascular or lymphatic or perineural invasion

${ }^{a}$ The control group of multivariate Cox analysis

Table 4 Toxicities of NACRT and complications of surgery

\begin{tabular}{ll}
\hline & No. (\%) \\
\hline Myelosuppression & \\
Grade 0-2 & $83(83.0 \%)$ \\
Grade 3-4 & $17(17.0 \%)$ \\
Mucositis/dermatitis & \\
Grade 0-2 & $97(97.0 \%)$ \\
Grade 3-4 & $3(3.0 \%)$ \\
Gl toxicities & \\
Grade 0-2 & $93(93.0 \%)$ \\
Grade 3-4 & $7(7.0 \%)$ \\
Intestinal obstruction & \\
Yes & $8(8.0 \%)$ \\
No & $92(92.0 \%)$ \\
Postsurgical complications & \\
Grade 0-2 & $82(92.1 \%)$ \\
Grade 3-4 & $7(7.9 \%)$ \\
Nonresectable surgery & 11 \\
Anastomotic leakage & $86(96.8 \%)$ \\
Yes & 11 \\
No &
\end{tabular}

Abbreviation: Gl Gastrointestinal

${ }^{\text {a Includes }} 7$ patients who abandoned surgery and 4 patients who underwent $\mathrm{EL}$
LARC [20]. The higher R0 resection rate of LACC in our study may translate to long-term survival benefit. The 3-year PFS and OS rates were 68.6 and $82.1 \%$, respectively, similar to two previous studies $[8,9]$, and significantly higher than rates reported in the literatures for patients undergoing neoadjuvant chemotherapy [21]. Recently, a research team has compared the treatment outcomes of cT4 colon cancers treated with neoadjuvant radiotherapy (NRT) to patients treated without NRT in their tertiary care center and the National Cancer Database [22, 23]. Both studies showed that the 5-year OS rate of the NRT group was 20 to $25 \%$ higher than that of the non-NRT group, and even when patients with cT4b tumors received NRT than non-NRT. Thus, the survival benefit of NACRT is plausible for patients with cT4 colon cancer.

Univariate and multivariate survival analyses revealed independent association of pathological grade with OS. The results were consistent with findings from the study by Wang el at, who constructed a prediction model to predict cause-specific death in elderly patients with colorectal cancer after surgery, particularly for patients with colon cancer [24]. By performing subgroup analysis, patients with better $\mathrm{T}$ downstaging had higher survival rates in the present study (Supplementary Figure 3E). Similar results were obtained from the analysis of ypTNM staging (Supplementary Figure 3G). Meanwhile, survival was prolonged in the patients who achieved pCR than in the patients who did not achieve pCR after 
NACRT. However, the difference was not statistically significant and may be caused by the small sample sizes.

The incidence of acute toxicities of NACRT is another important concern. Bone marrow toxicity was the most common adverse event, of which the incidence grade 34 adverse events was $17.0 \%$. The incidences of severe gastrointestinal and skin reactions were similar to patients who received neoadjuvant chemotherapy for LACC [25]. According to previous studies, IMRT accurately delivers radiation to tumors and decreases the dose administered to normal tissues [26]. In our study, 97 (97.0\%) patients successfully completed the full doses of radiotherapy. Three patients (3.0\%) experienced tumor perforation during the course of NACRT. We speculated that the tumor perforation may be attributed to the radiotherapy, which may cause rapid tumor regression. Therefore, based on the results of the present study, NACRT for colon cancer is safe and tolerable.

In Canadian studies, all patients underwent MVR with a relatively high incidence ( $>30 \%$ ) of postoperative complications [17, 18, 27]. The incidence of postsurgical complications in our series was lower, namely, 7.0\%. And no significant difference in survival existed between the MVR group and non-MVR group (Supplementary Fig 3L). Hence, we consider that NACRT can reduce the probability of MVR and decrease the incidence of postoperative complications.

As is well known that the preservation of bladder function is of vital importance to patients in terms of quality of life [28]. In our study, the bladder function was retained in $30.95 \%$ of patients with primary bladder invasion. Similarly, several adjacent organs avoided surgical resection or the scope of surgical resection was reduced, such as the small intestine, duodenum, kidney, liver, pancreas and large blood vessels. Therefore, this treatment strategy is more conducive to the preservation of organs and functions, with the results of an improvement in the survival rate and an improvement in the quality of life.

Sensitivity of the deficient mismatch repair (dMMR) phenotype to conventional chemotherapy and radiotherapy is still controversial [29]. At the ASCO conference in 2019, Matt et al. presented an oral report stating that the rate of tumor regression after neoadjuvant chemotherapy markedly was reduced in patient with $\mathrm{dMMR}$ tumors, while the rate of $\mathrm{pCR}$ was similar. In our study, patients with the dMMR phenotype had similar survival rates to patients with the pMMR phenotype $(p=0.880)$. Notably, dMMR tumors present opportunities for immunotherapy [30]. Further studies are needed to determine whether NACRT combined with immunotherapy improves the prognosis of patients with dMMR tumors, and we initiated a phase II clinical trial (NCT04301557).
The limitations of the study are the nature of nonrandomized study, moderate sample size and the follow-up time was not long enough. In addition, the specific strategies of NACRT for patients with unresectable LACC enrolled in our study were inconsistent. The optimal strategy of NACRT for LACC requires further investigation. Therefore, we are conducting an open multicenter, randomized controlled trial to further validate the results by comparing the efficacy of neoadjuvant chemotherapy and NACRT in patients with unresectable LACC (NCT03970694). To date, 25 patients have been recruited and the prospective data are expected, which may provide the higher levels of evidence.

In conclusion, NACRT might provide an opportunity for patients with unresectable LACC to achieve R0 resection, which might translate into a survival benefit and better quality of life.

\section{Supplementary Information}

The online version contains supplementary material available at https://doi. org/10.1186/s12885-021-07894-6.

Additional file 1: Supplementary Figure 1. The flowchart of treatment of unresectable LACC. Abbreviations: MVR, multivisceral resection; EL, exploratory laparotomy; CME, complete mesocolic excision. Supplementary Figure 2. The surgical details of adjacent organs. Bladder (A); Small intestine (B). Supplementary Figure 3. Subgroup analysis of survival. OS analyzed in patients with unresectable LACC treated with NACRT and surgery by differentiation (A), Resection group $(C)$, ypT stage (E), ypTNM stage $(G), M V R(L)$, TRG score $(M)$. PFS analyzed in all patients by differentiation (B), resection group (D), ypT stage (F), ypTNM stage $(H)$. DFS analyzed in patients with radical surgery by KPS (I), Differentiation (J), VPLNI (K). $P$ values in the figure were calculated from the comparison of the groups. NRS: Nonresectable surgery.

Supplementary Table 1. Tumor characteristics and treatment of patients who abandoned surgery. Supplementary Table 2. Characteristics of studies included in the discussion. Supplementary Table 3. Univariate Cox analysis of prognostic factors for OS, PFS, DFS. Supplementary Table 4. 1. Multiple linear regression coefficients. 2. Correlation matrix analysis.

\section{Abbreviations}

LACC: Locally advanced colon cancer; NACRT: Neoadjuvant

chemoradiotherapy; MVR: Multivisceral resection; MDT: Multidisciplinary team; AJCC: American Joint Cancer Committee; CT: Computed tomography; MRI: Magnetic resonance image; CEA: Carcinoembryonic antigen; CME: Complete mesocolic excision; CTCAE: Common Terminology Criteria for Adverse Events; IQRs: Interquartile ranges; PFS: Progression-free survival; OS: Overall survival; DFS: Disease-free survival; LR: Local recurrence; DM: Distant metastasis; Cl: Confidence interval; Gl: Gastrointestinal; NRT: Neoadjuvant radiotherapy; dMMR: Deficient mismatch repair

\section{Acknowledgments}

We thank all the clinicians and patients who participated in the study.

\section{Authors' contributions}

R.Z. and Y.H.G. had the original idea for the study. Y.H.G. had access to all data in the study, and were responsible for the integrity of the data and the accuracy of the data analyses. Y.Y., W.W.X. and W.H.X. participated in analysing the data and writing of the manuscript. P.Q.C. reviewed radiologic images of all the patients. Y.H.G., Q.X.W., H.C. and B.Q.C. were involved in the revision of the manuscript. Y.H.G., W.H.Z., Z.F.Z., X.J.W. and L.R.L. were involved in the design of the study and collected the data. Q.L. was 
responsible for the accuracy of the statistical analysis. All authors have read, edited, and approved the final version of the manuscript.

\section{Funding}

This work was supported by National Natural Science Foundation of China (81672987; 82073329), Natural Science Foundation of Guangdong Province (2020A1515011286).

\section{Availability of data and materials}

All data are available via the corresponding author.

\section{Ethics approval and consent to participate}

This observational study was performed in accordance with the Declaration of Helsinki and approved by the Medical Ethics Committee of Sun Yat-sen University Cancer Center (B2020-063-01). All patients have signed the informed consent form before treatment.

\section{Consent for publication}

Not applicable.

\section{Competing interests}

The authors report no conflicts of interest.

\section{Author details}

${ }^{1}$ State Key Laboratory of Oncology in South China, Collaborative Innovation Center for Cancer Medicine, Guangzhou, China. ${ }^{2}$ Department of Radiation Oncology, Sun Yat-sen University Cancer Center, Guangzhou, China. ${ }^{3}$ Departments of Medical Imaging and Interventional Radiology, Sun Yat-sen University Cancer Center, Guangzhou, PR China. ${ }^{4}$ Department of Colorectal Surgery, Sun Yat-sen University Cancer Center, Guangzhou, PR China. ${ }^{5}$ Department of Epidemiology and Biostatistics, Sun Yat-sen University Cancer Center, Guangzhou, PR China. 'Department of Endoscopy and Laser, Sun Yat-sen University Cancer Center, Guangzhou, PR China.

\section{Received: 17 December 2020 Accepted: 5 February 2021}

\section{Published online: 19 February 2021}

\section{References}

1. Bray F, Ferlay J, Soerjomataram I, Siegel RL, Torre LA, Jemal A. Global cancer statistics 2018: GLOBOCAN estimates of incidence and mortality worldwide for 36 cancers in 185 countries. CA Cancer J Clin. 2018;68(6):394-424.

2. van der Geest LG, Lam-Boer J, Koopman M, Verhoef C, Elferink MA, de Wilt $\mathrm{JH}$. Nationwide trends in incidence, treatment and survival of colorectal cancer patients with synchronous metastases. Clin Exp Metastasis. 2015: 32(5):457-65.

3. Schmoll HJ, Van Cutsem E, Stein A, Valentini V, Glimelius B, Haustermans K, Nordlinger B, van de Velde CJ, Balmana J, Regula J, et al. ESMO consensus guidelines for management of patients with colon and rectal cancer. A personalized approach to clinical decision making. Ann Oncol. 2012;23(10): 2479-516.

4. Leijssen LGJ, Dinaux AM, Amri R, Kunitake H, Bordeianou LG, Berger DL. The impact of a multivisceral resection and adjuvant therapy in locally advanced colon cancer. J Gastrointest Surg. 2019;23(2):357-66.

5. Khan MA, Hakeem AR, Scott N, Saunders RN. Significance of R1 resection margin in colon cancer resections in the modern era. Color Dis. 2015;17(11): 943-53.

6. Sauer R, Liersch T, Merkel S, Fietkau R, Hohenberger W, Hess C, Becker H, Raab HR, Villanueva MT, Witzigmann $\mathrm{H}$, et al. Preoperative versus postoperative chemoradiotherapy for locally advanced rectal cancer: results of the German CAO/ARO/AIO-94 randomized phase III trial after a median follow-up of 11 years. J Clin Oncol. 2012;30(16):1926-33.

7. Agas RAF, Co LBA, Sogono PG, Jacinto J, Yu KKL, Jacomina LE, Bacorro WR, Sy Ortin $\Pi$. Assessing the effect of radiotherapy in addition to surgery in colon adenocarcinomas: a systematic review and meta-analysis of contemporary evidence. J Gastrointest Cancer. 2019;51:445-60.

8. Chang H, Yu X, Xiao W, Wang Q, Zhou W, Zeng Z, Ding P, Li L, Gao Y. Neoadjuvant chemoradiotherapy followed by surgery in patients with unresectable locally advanced colon cancer: a prospective observational study. Onco Targets Ther. 2018;11:409-18.

9. Qiu B, Ding P, Cai L, Xiao W, Zeng Z, Chen G, Lu Z, Li L, Wu X, Mirimanoff R, et al. Outcomes of preoperative chemoradiotherapy followed by surgery in patients with unresectable locally advanced sigmoid colon cancer. Chin J Cancer. 2016:35(1):65.

10. Charlson ME, Pompei $P$, Ales KL, Mackenzie CR. A new method of classifying prognostic comorbidity in longitudinal studies development and validation. J Chron Dis. 1987;40(5):373-83.

11. Dindo D, Demartines N, Clavien PA. Classification of surgical complications: a new proposal with evaluation in a cohort of 6336 patients and results of a survey. Ann Surg. 2004;240(2):205-13.

12. Tie J, Cohen JD, Wang Y, Christie M, Simons K, Lee M, Wong R, Kosmider S, Ananda S, McKendrick J, et al. Circulating tumor DNA analyses as markers of recurrence risk and benefit of adjuvant therapy for stage III colon cancer. JAMA Oncol. 2019;5(12):1710-7.

13. NCCN Clinical Practice Guidelines in Oncology (NCCN Guidelines ${ }^{\circledR}$ ):Colon Cancer (version 1. 2020). NCCN.org. Available from: https://www.nccn.org/ store/login/login.aspx?. Accessed 19 Dec 2019.

14. Mizukami H, Yoshizawa Y, Sasaya S, Nemoto H, Maezawa K, Sanada Y. A case of advanced colon cancer invading the rectum effectively treated with chemoradiation therapy before surgery. Gan To Kagaku Ryoho Cancer Chemother. 2007:34(6):953-6.

15. Kuga Y, Tanaka T, Arita M, Okanobu H, Miwata T, Yoshimi S, Murakami E, Numata Y, Moriya T, Ohya T, et al. A case of effective chemotherapy using S-1 and CPT-11 following chemoradiotherapy with UFT and Leucovorin for unresectable advanced sigmoid colon cancer. Gan To Kagaku Ryoho Cancer Chemother. 2010;37(3):531-4.

16. Taylor WE, Donohue JH, Gunderson LL, Nelson H, Nagorney DM, Devine RM, Haddock MG, Larson DR, Rubin J, O'Connell MJ. The Mayo Clinic experience with multimodality treatment of locally advanced or recurrent colon cancer. Ann Surg Oncol. 2002;9(2):177-85.

17. Cukier M, Smith AJ, Milot L, Chu W, Chung H, Fenech D, Herschorn S,

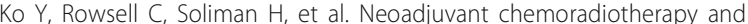
multivisceral resection for primary locally advanced adherent colon cancer: a single institution experience. Eur J Surg Oncol. 2012;38(8):677-82

18. Hallet J, Zih FS, Lemke M, Milot L, Smith AJ, Wong CS. Neo-adjuvant chemoradiotherapy and multivisceral resection to optimize R0 resection of locally recurrent adherent colon cancer. Eur J Surg Oncol. 2014:40(6):706-12.

19. Huang CM, Huang MY, Ma CJ, Yeh Y, Tsai HL, Huang CW, Huang CJ, Wang JY. Neoadjuvant FOLFOX chemotherapy combined with radiotherapy followed by radical resection in patients with locally advanced colon cancer. Radiat Oncol. 2017;12(1):48

20. Hu YH, Wei JW, Chang H, Xiao W, Lin JZ, Cai MY, Cai PQ, Kong LH, Chen G, Pan $Z Z$, et al. The high $\mathrm{pCR}$ rate of sandwich neoadjuvant treatment in locally advanced rectal cancer may translate into a better long-term survival benefit: 5-year outcome of a phase II clinical trial. Cancer Manag Res. 2018; 10:4363-9.

21. Dehal A, Graff-Baker AN, Vuong B, Fischer T, Klempner SJ, Chang S-C, Grunkemeier GL, Bilchik AJ, Goldfarb M. Neoadjuvant chemotherapy improves survival in patients with clinical T4b colon cancer. J Gastrointest Surg. 2017:22(2):242-9.

22. Krishnamurty DM, Hawkins AT, Wells KO, Mutch MG, Silviera ML, Glasgow SC, Hunt SR, Dharmarajan S. Neoadjuvant radiation therapy in locally advanced colon cancer: a cohort analysis. J Gastrointest Surg. 2018;22(5): 906-12.

23. Hawkins AT, Ford MM, Geiger TM, Hopkins MB, Kachnic LA, Muldoon RL, Glasgow SC. Neoadjuvant radiation for clinical T4 colon cancer: a potential improvement to overall survival. Surgery. 2019;165(2):469-75.

24. Wang Z, Wang Y, Yang Y, Luo Y, Liu J, Xu Y, Liu X. A competing-risk nomogram to predict cause-specific death in elderly patients with colorectal cancer after surgery (especially for colon cancer). World J Surg Oncol. 2020;18(1):30

25. Jakobsen A, Andersen F, Fischer A, Jensen LH, Jorgensen JC, Larsen O Lindebjerg J, Ploen J, Rafaelsen SR, Vilandt J. Neoadjuvant chemotherapy in locally advanced colon cancer. A phase II trial. Acta Oncol. 2015;54(10): 1747-53.

26. Wieland P, Dobler B, Mai S, Hermann B, Tiefenbacher U, Steil V, Wenz F, Lohr F. IMRT for postoperative treatment of gastric cancer: covering large target volumes in the upper abdomen: a comparison of a stepand-shoot and an arc therapy approach. Int J Radiat Oncol Biol Phys. 2004;59(4):1236-44

27. Lehnert T, Methner M, Pollok A. Multivisceral resection for locally advanced primary colon and rectal cancer. Ann Surg. 2002;235(2):217-25. 
28. Li YL, Wen JJ, Wen YB, He XF, Wu JW, Li YW, Han ZJ, Feng JJ, Yan SH, Li SL, et al. Reconstruction of bladder function and prevention of renal deterioration by means of end-to-side neurorrhaphy in rats with neurogenic bladder. Neurourol Urodyn. 2018;37(4):1272-80.

29. Zaanan A, Shi Q, Taieb J, Alberts SR, Meyers JP, Smyrk TC, Julie C, Zawadi A, Tabernero J, Mini E, et al. Role of deficient DNA mismatch repair status in patients with stage III colon cancer treated with FOLFOX adjuvant chemotherapy: a pooled analysis from 2 randomized clinical trials. JAMA Oncol. 2018:4(3):379-83.

30. Ganesh K, Stadler ZK, Cercek A, Mendelsohn RB, Shia J, Segal NH, Diaz LA. Immunotherapy in colorectal cancer: rationale, challenges and potential. Nat Rev Gastroenterol Hepatol. 2019;16(6):361-75.

\section{Publisher's Note}

Springer Nature remains neutral with regard to jurisdictional claims in published maps and institutional affiliations.

Ready to submit your research? Choose BMC and benefit from:

- fast, convenient online submission

- thorough peer review by experienced researchers in your field

- rapid publication on acceptance

- support for research data, including large and complex data types

- gold Open Access which fosters wider collaboration and increased citations

- maximum visibility for your research: over $100 \mathrm{M}$ website views per year

At BMC, research is always in progress.

Learn more biomedcentral.com/submissions 